\title{
Application of Fixed Point Theorems in Fuzzy Metric Spaces for Implicit Relation
}

\author{
M.Vijaya Kumar ${ }^{1}$,S.M.Subhani ${ }^{2}$
}

\begin{abstract}
In this paper we prove a common fixed point theorem in fuzzy metric space using an implicit relation. 2010 AMS Classification: 47H10, 54H25

Keywords: Fuzzy metric space, Fixed point, Fixed point Theorem, Compatible mapping, Reciprocal continuity.
\end{abstract}

\section{Introduction}

Zadeh [13] introduced the notion of fuzzy sets In 1965. presently many authors have widely developed the theory of fuzzy sets and their application. The suggestion of fuzzy metric space introduced by Kramosil and Michalek [4] was adapted by George and Veeramani [3]. In [8] Popa proved theorem for weakly compatible continuous mappings using implicit relation. Singh et. al. [10] introduced the notion of compatible maps in fuzzy metric space, and compared this notion of compatible map, compatible map of type ( $\beta$ ), compatible map of type $(\alpha)$, and get some fixed point theorems in absolute fuzzy metric space in the sense of Grabiec [2]. In [12] Vasuki introduce the concept of R-weakly commuting map, and prove a fixed point theorem in fuzzy metric space. Ranadive et. al. [9] introduced the concept of metric space and proves the common fixed point theorem in this space. Moreover Ranadive et. al. [9] observed that the new notion of map is neither a subclass of compatible maps nor a subclass of non-compatible maps. In [6] Mishra et. al. introduced maps in fuzzy metric space. In this paper, we obtain fixed point theorems in fuzzy metric space using maps, an implicit relation with reciprocal continuity and compatible maps.

\section{Preliminaries}

We introduce some definitions and known results in fuzzy metric space.

Definition 2.1 ( [13]). Let fuzzy set $A$ in $X$ is a function with domain $X$ and value in $[0,1]$.

Definition 2.2 ( [11]). A triangular norm * (shortly t-norm) is a binary operation on the unit interval [0,1] such that for all $\mathrm{a} ; \mathrm{b} ; \mathrm{c} ; \mathrm{d} \in[0,1]$ the following conditions are fulfilled :

(1) $\mathrm{a} * 1=1$,

(2) $a * b=b * a$,

(3) $\mathrm{a} * \mathrm{~b} \cdot \mathrm{c} * \mathrm{~d}$ whenever $\mathrm{a} \leq \mathrm{c}$ and $\mathrm{b} \leq \mathrm{d}$,

(4) $\mathrm{a} *(\mathrm{~b} * \mathrm{c})=(\mathrm{a} * \mathrm{~b}) * \mathrm{c}$.

Definition 2.3 ( [4]). The 3-tuple (X;M; *) is called a fuzzy metric space if $\mathrm{X}$ is an arbitrary non-empty set, * is a continuous $\mathrm{t}-$ norm and $\mathrm{M}$ is a fuzzy set in $\mathrm{X}^{2} \mathrm{x}[0, \infty)$ satisfying the following conditions, for all $\mathrm{x}, \mathrm{y}, \mathrm{z} \in \mathrm{X}$ and $\mathrm{s}, \mathrm{t}>0$.

(1) $\mathrm{M}(\mathrm{x}, \mathrm{y}, 0)=0$;

(2) $M(x, y, t)=1$ for all $t>0$, iff $x=y$,

(3) $\mathrm{M}(\mathrm{x}, \mathrm{y}, \mathrm{t})=\mathrm{M}(\mathrm{y}, \mathrm{x}, \mathrm{t})$,

(4) $M(x, y, t) * M(y, z, s), M(x, z, t+s)$,

(5) $\mathrm{M}(\mathrm{x}, \mathrm{y},):.[0, \infty) \rightarrow[0,1]$ is left continuous ;

(6) $\lim _{\mathrm{t} \rightarrow \infty} \mathrm{M}(\mathrm{x}, \mathrm{y}, \mathrm{t})=1$.

In the definition of George and Veeramani [3] $M$ is a fuzzy set on $X^{2} x[0, \infty)$ an(1), (2), (5) are replaced respectively, with ( I), ( II), (III) below :

(I) $\quad \mathrm{M}(\mathrm{x}, \mathrm{y}, 0)>0$, for all $\mathrm{t}>0$,

(II) $\quad \mathrm{M}(\mathrm{x}, \mathrm{x}, \mathrm{t})=1$, for all $\mathrm{t}>0$ and $\mathrm{x}=\mathrm{y}) \mathrm{M}(\mathrm{x}, \mathrm{y}, \mathrm{t})<1$ for all $\mathrm{t}>0$,

(III) $\quad \mathrm{M}(\mathrm{x}, \mathrm{y},):.(0, \infty) \rightarrow[0,1]$ is continuous for all $\mathrm{x}, \mathrm{y} \in \mathrm{X}$.

Example 2.4 ( [3]). Let $(X, d)$ be a metric space. Define $a * b=a b$ (or $a * b=\min [a, b]$ ) and for all $x, y \in X$ and $\mathrm{t}>0, \mathrm{M}(\mathrm{x}, \mathrm{y}, \mathrm{t})=\mathrm{t} / \mathrm{t}+\mathrm{d}(\mathrm{x}, \mathrm{y})$.

Then $(\mathrm{X}, \mathrm{M}, *)$ is a fuzzy metric space. We identify this is fuzzy metric $\mathrm{M}$ induced by the metric $\mathrm{d}$ the standard fuzzy metric.

Definition 2.5 ( [3]). A sequence $\left\{x_{n}\right\}$ in a fuzzy metric space $\left(X, M,{ }^{*}\right)$ is called 
1. Cauchy if for each $\varepsilon>0$ and $\mathrm{t}>0$, there exists $n_{0} \in N$ such that $\mathrm{M}\left(\mathrm{x}_{\mathrm{n}}, \mathrm{x}_{\mathrm{m}}, \mathrm{t}\right)>1-\varepsilon$ for all $\mathrm{n}, \mathrm{m} \geq \mathrm{n}_{0}$.

2. Complete if every Cauchy sequence in $X$ converges to a point in $X$.

3. Convergent to $\mathrm{x} \in \mathrm{X}$ if $\lim _{\mathrm{n} \rightarrow \infty} \mathrm{M}\left(\mathrm{x}_{\mathrm{n}}, \mathrm{x}, \mathrm{t}\right)>1-\varepsilon$ for each $\mathrm{t}>0$, there exists $n_{0} \in N$.

Definition 2.6 ( [1]). A pair (A, B) of self maps of a fuzzy metric space (X, M, *) is said to 1.reciprocal continuous if $\lim _{n \rightarrow \infty} A B x_{n}=A x$ and $\lim _{n \rightarrow \infty} B A x_{n}=B x$, whenever there exists a sequence $\left\{x_{n}\right\} \in X$ such that $\lim _{n \rightarrow \infty} A x_{n}=\lim _{n \rightarrow \infty} B x_{n}=x$ for some $x \in X$. If $A$ and $B$ are both continuous

2.semi-compatible if $\lim _{n \rightarrow \infty} A B x_{n}=B x$ whenever there exists a sequence $\left\{x_{n}\right\} \in X$ such that $\lim _{n \rightarrow \infty} A x_{n}=$ $\lim _{n \rightarrow \infty} \mathrm{Bx}_{\mathrm{n}}=\mathrm{x}$ for some $\mathrm{x} \in \mathrm{X}$.

Note: then they are obviously reciprocally continuous but the converse need not be true.

Lemma 2.7 ( [5]). Let $(X, M, *)$ be a fuzzy metric space. If there exists $k \in(0,1)$ such that for all $\mathrm{x}, \mathrm{y} \in \mathrm{X}, \mathrm{t}>0$ ,$M(x, y, k t) \geq M(x, y, t)$, then $x=y$.

Lemma $2.8([2])$. $M(x, y,$.$) is non-decreasing for all x, y \in X$.

Proposition 2.9 ([7]). Let A and B be two self maps on a fuzzy metric space (X, M, *). Assume that (A, B) is reciprocal continuous then $(\mathrm{A}, \mathrm{B})$ is semi-compatible if and only if $(\mathrm{A}, \mathrm{B})$ is compatible.

\section{A class of implicit relation}

Let $\Phi$ be the set of all real continuous functions $F:(R+)^{5} \rightarrow R$ non-decreasing in first argument satisfying the following conditions :

(i) For $\mathrm{u}, \mathrm{v} \geq 0, \mathrm{~F}(\mathrm{u}, \mathrm{v}, \mathrm{v}, \mathrm{u}, 1) \geq 0$ implies that $\mathrm{u} \geq \mathrm{v}$.

(ii) $\mathrm{F}(\mathrm{u}, 1,1, \mathrm{u}, 1) \geq 0$ or $\mathrm{F}(\mathrm{u}, 1, \mathrm{u}, 1, \mathrm{u}) \geq 0$, or $\mathrm{F}(\mathrm{u}, \mathrm{u}, 1,1, \mathrm{u}) \geq 0$ implies that $\mathrm{u} \geq 1$.

Proof. For $\mathrm{u}, \mathrm{v} \geq 0$;

(i) $\mathrm{F}(\mathrm{u}, \mathrm{v}, \mathrm{v}, \mathrm{u}, 1) \geq 0=(\mathrm{a}+\mathrm{d}) \mathrm{u}+(\mathrm{b}+\mathrm{c}) \mathrm{v} \geq 0$,

$=>(a+d) u \geq(a+d) v$, i.e. $u \geq v$,

(ii) $\mathrm{F}(\mathrm{u}, 1,1, \mathrm{u}, 1) \geq 0=(\mathrm{a}+\mathrm{d}) \mathrm{u}+(\mathrm{b}+\mathrm{c}) \geq 0$,

$=(a+d) u \geq(a+d)$, i.e. $u \geq 1$

Example 3.1. Define $\mathrm{F}\left(\mathrm{t}_{1}, \mathrm{t}_{2}, \mathrm{t}_{3}, \mathrm{t}_{4}, \mathrm{t}_{5}\right)=10 \mathrm{t}_{1}-8 \mathrm{t}_{2}-7 \mathrm{t}_{3}+5 \mathrm{t}_{4}+\mathrm{t}_{5}-1$. Then $F \in \Phi$.

(i) $\mathrm{F}(\mathrm{u}, \mathrm{v}, \mathrm{v}, \mathrm{u}, 1)=15(\mathrm{u}-\mathrm{v}) \geq 0 \Rightarrow \mathrm{u} \geq \mathrm{v}$.

(ii) $\mathrm{F}(\mathrm{u}, 1,1, \mathrm{u}, 1)=15(\mathrm{u}-1) \geq 0 \Rightarrow \mathrm{u} \geq 1$ or

$\mathrm{F}(\mathrm{u}, 1, \mathrm{u}, 1, \mathrm{u})=4(\mathrm{u}-1) \geq 0=>\mathrm{u} \geq 1$

or

$\mathrm{F}(\mathrm{u}, \mathrm{u}, 1,1, \mathrm{u})=\mathrm{u} \geq 1$

A characterization of $\Phi$ in linear form : Define

$\mathrm{F}\left(\mathrm{t}_{1}, \mathrm{t}_{2}, \mathrm{t}_{3}, \mathrm{t}_{4}, \mathrm{t}_{5}\right)=\mathrm{at}_{1}+\mathrm{bt}_{2}+\mathrm{ct}_{3}+\mathrm{dt}_{4}+\mathrm{t}_{5}-1$,

where $a, b, c, d \in R$, with $a+b+c+d=0, a>0, b+d>0, c+d>0$, and $a+d>0$.

Then $F \in \Phi$.

\section{Main result}

Theorem4.1. Let A, B, S and T be self mappings of a complete fuzzy metric space (X, M, *) with $\mathrm{t}$ - norm defined by $\mathrm{a} * \mathrm{~b}=\min \{\mathrm{a} \mathrm{b}\}$, satisfying :

$(4.1,1) \quad A(X) \subseteq T(X), B(X) \subseteq S(X)$

(4.1.2) for some $F \in \Phi$, there exists $\mathrm{k} \in(0,1)$ such that for all $\mathrm{x}, \mathrm{y} \in \mathrm{X}$ and $\mathrm{t}>0$,

$$
\left\{\begin{array}{c}
{\left[F_{A x, B y}(k u)\right]^{2},\left[F_{S x}, T y(u)^{2}\right] F_{S x, T y}(u) F_{T y, B y}(u), F_{S x, T y}(u) F_{S x, A x}(u),} \\
F_{S x, T y}(u) F_{T y, B y}(u), F_{S x, T y}(u) F_{S x, B y}(2 u), F_{S x, T y}(u) F_{T y, A x}(u), \\
F_{S x, B y}(2 u) F_{T y, A x}(u), F_{S x, A x}(u) F_{T y, A x}(u), F_{S x, B y}(2 u) F_{T y, B y}(u)
\end{array}\right\} \geq 0
$$

If the pair $\{\mathrm{A}, \mathrm{S}\}$ is reciprocal continuous Then $\mathrm{A}, \mathrm{B}, \mathrm{S}$ and $\mathrm{T}$ have a unique common fixed point in $\mathrm{X}$.

Proof. Since $\mathrm{A}(\mathrm{X}) \subset \mathrm{T}(\mathrm{X})$ for any arbitrary $x_{0} \in X$, there exists a point $\mathrm{x}_{1} \in \mathrm{X}$ such that $\mathrm{Ax}_{0}=\mathrm{Tx}_{1}$ and $\mathrm{B}(\mathrm{X}) \subset \mathrm{S}(\mathrm{X})$ implies that for this point $\mathrm{x}_{1}$ we can find a point $\mathrm{x}_{2} \in \mathrm{X}$ such that $\mathrm{Bx}_{1}=\mathrm{Sx}_{2}$ and so on. Inductively, we can define a sequence $\left\{\mathrm{y}_{\mathrm{n}}\right\}$ in $\mathrm{X}$ such that

$$
\mathrm{y}_{2 \mathrm{n}}=\mathrm{Ax}_{2 \mathrm{n}}=\mathrm{Tx}_{2 \mathrm{n}+1}
$$




$$
\mathrm{y}_{2 \mathrm{n}+1}=\mathrm{Bx}_{2 \mathrm{n}+1}=\mathrm{Sx}_{2 \mathrm{n}+2}, \quad \mathrm{n}=0,1,2, \ldots \ldots, \ldots
$$

Now we prove that the sequence defined by (2.7) is a Cauchy sequence. By Lemma (2.8) it is sufficient to show that $F_{y 2 n, y 2 n+1}(k u) \geq F_{y 2 n-1, y 2 n}(u)$ for all $u>0$ where $k \in(0,1)$. Suppose that $F_{y 2 n, y 2 n+1}(k u)<F_{y 2 n-1, y 2 n}(u)$ and using (4.1.2), we have

$$
\begin{aligned}
& \left(\left[F_{y_{2 n}, y_{2 n+1}}(k u)\right]^{2}=\left[F_{A x_{2 n}, B x_{2 n+1}}(k u)\right]^{2},\left[F_{S x_{2 n}, T x_{2 n+1}}(u)\right]^{2}, F_{S x_{x_{n},}, A x_{2 n}}(u) F_{T_{x_{2 n+1}, B x_{2 n+1}}}(u),\right. \\
& F_{S x_{2 n}, T x_{2 n+1}}(u) F_{S x_{2 n}, A x_{2 n}}(u), F_{S x_{x_{n} n}, T x_{2 n+1}}(u) F_{T x_{2 n+1}, B x_{2 n+1}}(u), F_{S x_{2 n}, T X_{2 n+1}}(u) F_{S x_{2 n}, B x_{2 n+1}}(2 u),\{0 \\
& F_{S x_{2 n}, T X_{2 n+1}}(u) F_{T x_{2 n}, A x_{2 n}}(u), F_{S x_{x_{2 n}, B x_{2 n+1}}}(2 u) F_{T x_{2 n+1}, A x_{2 n}}(u), F_{S x_{x_{2 n}}, A x_{2 n}}(u) F_{T x_{x_{n n+}}, A x_{2 n}}(u), \\
& F_{S x_{2 n}, B x_{2 n+1}}(2 u) F_{T x_{2 n+1}, B x_{2 n+1}}(u) \\
& \left\{\begin{array}{l}
{\left[F_{y 2 n-1, y 2 n}(u)\right]^{2}, F_{y 2 n-1, y 2 n}(u) F_{y 2 n, y 2 n+1}(u), F_{y 2 n-1, y 2 n}(u) F_{y 2 n-1, y 2 n}(u),} \\
F_{y 2 n-1, y 2 n}(u) F_{y 2 n, y 2 n+1}(u), F_{y 2 n-1, y 2 n}(u) F_{y 2 n-1, y 2 n+1}(2 u), F_{y 2 n-1, y 2 n}(u) F_{y 2 n, y 2 n}(u), \\
F_{y 2 n-1, y 2 n+1}(2 u) F_{y 2 n, y 2 n}(u), F_{y 2 n-1, y 2 n}(u) F_{y 2 n, y 2 n}(u), F_{y 2 n-1, y 2 n+1}(2 u) F_{y 2 n, y 2 n+1}(u)
\end{array}\right\} \geq 0 \\
& \left\{\begin{array}{l}
{\left[F_{y 2 n, y 2 n+1}(k u)\right]^{2}, F_{y 2 n, y 2 n+1}(k u) F_{y 2 n, y 2 n+1}(u),\left[F_{y 2 n, y 2 n+1}(k u)\right]^{2}, F_{y 2 n, y 2 n+1}(k u)} \\
F_{y 2 n, y 2 n+1}(u), F_{y 2 n, y 2 n+1}(k u) t\left\{F_{y 2 n-1, y 2 n}(u) F_{y 2 n, y 2 n+1}(u)\right\}, F_{y 2 n, y 2 n+1}(k u), \\
t\left\{F_{y 2 n-1, y 2 n}(u), F_{y 2 n, y 2 n+1}(u)\right\}, F_{y 2 n, y 2 n+1}(k u), t\left\{F_{y 2 n-1, y 2 n}(u) F_{y 2 n, y 2 n+1}(u)\right\} F_{y 2 n, y 2 n+1}(u)
\end{array}\right\} \geq 0
\end{aligned}
$$

Since $\mathrm{k} \in(0,1)$ then $\mathrm{u}>\mathrm{ku}$ for any $\mathrm{u}>0$ and $\mathrm{F}_{\mathrm{y} 2 \mathrm{n}, \mathrm{y} 2 \mathrm{n}+1}(\mathrm{u})>\mathrm{F}_{\mathrm{y} 2 \mathrm{n}, \mathrm{y} 2 \mathrm{n}+1}(\mathrm{ku})$ and also

$$
\begin{gathered}
t\left\{F_{y 2 n-1, y 2 n}(u), F_{y 2 n, y 2 n+1}(u)\right\} \geq t\left\{F_{y 2 n, y 2 n+1}(k u), F_{y 2 n, y 2 n+1}(u)\right\} \geq \\
t\left\{F_{y 2 n, y 2 n+1}(k u), F_{y 2 n, y 2 n+1}(k u)\right\} \geq F_{y 2 n, y 2 n+1}(k u)
\end{gathered}
$$

Hence,

$$
\left\{F_{y 2 n, y 2 n+1}\right\}(u),\left\{\left[F_{y 2 n, y 2 n+1}(k u)\right]^{2}, F_{y 2 n, y 2 n+1}(k u)\right\} \geq 0
$$

Since, $(X, F, t)$ is complete, then the sequence $\left\{y_{n}\right\}$ converges to point $z$ in $X$ and the subsequence $\left\{\mathrm{Ax}_{2 \mathrm{n}}\right\},\left\{\mathrm{Bx}_{2 \mathrm{n}+1}\right\},\left\{\mathrm{Sx}_{2 \mathrm{n}}\right\},\left\{\mathrm{Tx}_{2 \mathrm{n}+1}\right\}$ of $\left\{\mathrm{y}_{2 \mathrm{n}}\right\}$ also converge to $\mathrm{z}$.

Since $A(x) \subset B(x)$, there exists $\mathrm{p} \in \mathrm{X}$, such that $\mathrm{z}=$ Tp by using (3.3.3) we have

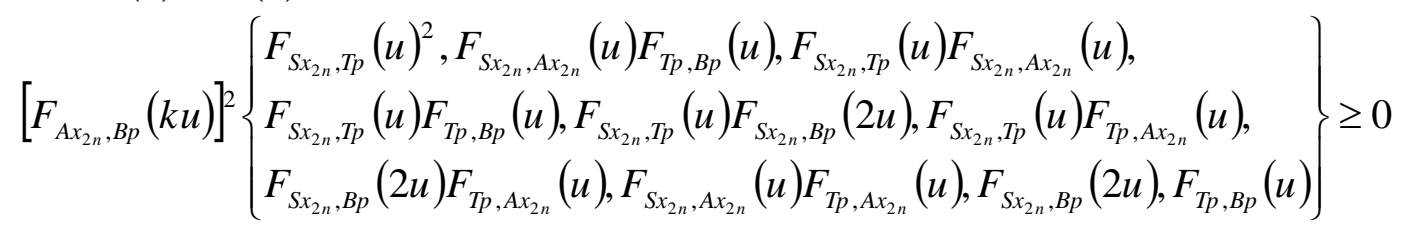

Taking limit as $\mathrm{n} \rightarrow \infty$ gives,

$$
\left\{\begin{array}{c}
{\left[F_{z, B p}(k u)\right]^{2},\left[F_{z, z}(u)\right]^{2}, F_{z, z}(u) F_{z, B p}(u), F_{z, z}(u) F_{z, z}(u), F_{z, z}(u) F_{z, B p}(u),} \\
\\
\left.F_{z, z}(u) F_{z, B p}(2 u), F_{z, z}(u) F_{z, z}(u), F_{z, B p}(2 u) F_{z, z}(u), F_{z, z}(u) F_{z, z}(u),\right\} \geq 0 \\
\\
F_{z, B p}(2 u) F_{z, B p}(u) \\
\left\{\left[F_{z, B p}(u)\right]^{2}, F_{z, B p}(u)\right\},\left[F_{z, B p}(u)\right]^{2} \geq 0
\end{array}\right.
$$

Which means that $\mathrm{Bp}=\mathrm{z}$ then we have $\mathrm{B}=\mathrm{Tp}=\mathrm{z}$.

By a similar way, since $B(X) \subset S(X)$, there exists $q \in X$ such that $\mathrm{z}=\mathrm{Sq}$.

Again by using (4.1.2).

$$
\left\{\begin{array}{c}
{\left[F_{A q, B \times 2 n+1}(k u)\right]^{2},\left[F_{S q, T \times 2 n+1}(u)\right]^{2}, F_{S q, A q}(u) F_{T \times 2 n+1, B \times 2 n+1}(u), F_{S q, T \times 2 n+1}(u) F_{S q, A q}(u)} \\
F_{S q, T x 2 n+1}(u) F_{T x 2 n+1, B \times 2 n+1}(u), F_{S q, T \times 2 n+1}(u) F_{S q, B x 2 n+1}(2 u), \\
F_{S q, T x 2 n+1}(u) F_{T x 2 n+1, A q}(u), F_{S q, B \times 2 n+1}(2 u) F_{T x 2 n+1, B x 2 n+1}(u)
\end{array}\right\} \geq 0
$$

Taking limit as $\mathrm{n} \rightarrow \infty$ gives, 
$\left\{\begin{array}{c}{\left[F_{A q, z}(k u)\right]^{2},\left[F_{z, z}(u)\right]^{2}, F_{z, A q}(u) F_{z, z}(u), F_{z, z}(u) F_{z, A q}(u), F_{z, z}(u) F_{z, z}(u),} \\ F_{z, z}(u) F_{z, z}(2 u), F_{z, z}(u) F_{z, A q}(u), F_{z, z}(2 u) F_{z, A q}(u), F_{z, A q}(u) F_{z, A q}(u) \\ F_{z, z}(2 u) F_{z, z}(u)\end{array}\right\} \geq 0$

$\left\{\left[F_{z, a q}(u)\right]^{2}, F_{z, A q}(u)\right\} \geq\left[F_{z, A q}(u)\right]^{2} \geq 0$

This yields $\mathrm{Aq}=\mathrm{z}$ then we have, $\mathrm{Aq}=\mathrm{Sq}=\mathrm{z}$.

$\mathrm{Bz}=\mathrm{Tz}$

Since $\{\mathrm{B}, \mathrm{T}\}$ are weakly compatible then they commute at their coincidence point $\mathrm{p}$. i.e., $\mathrm{BTp}=\mathrm{TBp}$ or

Now we show that $\mathrm{z}$ is a fixed point of $\mathrm{B}$.

By using (4.1.2),

$$
\left\{\begin{array}{c}
{\left[F_{A q, B z}(k u)\right]^{2},\left[F_{S q, T z}(u)\right]^{2}, F_{S q, A q}(u) F_{T z, B z}(u), F_{S q, T z}(u) F_{S q, A q}(u), F_{S q, T z}(u) F_{T z, B z}(u)} \\
\left.F_{S q, T z}(u) F_{S q, B z}(2 u), F_{S q, T z}(u) F_{T z, A q}(u), F_{S q, B z}(2 u) F_{T z, A q}(u), F_{S q, A q}(u) F_{T z, A q}(u)\right\} \geq 0 \\
F_{S q, B z}(2 u) F_{T z, B z}(u)
\end{array}\right\} \begin{gathered}
{\left[F_{z, B z}(k u)\right]^{2},\left[F_{z, B z}(u)\right]^{2}, F_{z, z}(u) F_{B z, B z}(u), F_{z, B z}(u) F_{z, z}(u), F_{z, B z}(u) F_{B z, B z}(u)} \\
\left.F_{z, B z}(u) F_{z, B z}(2 u), F_{z, B z}(u) F_{B z, z}(u), F_{z, B z}(2 u) F_{B z, z}(u), F_{z, z}(u) F_{B z, z}(u),\right\} \geq 0 \\
F_{z, B z}(2 u) F_{B z, B z}(u) \\
\left\{\left[F_{z, B z}(u)\right]^{2}, F_{z, B z}(u)\right\} \geq 0 \\
{\left[F_{z, B z}(u)\right]^{2} \geq 0}
\end{gathered}
$$

Which implies that $\mathrm{Bz}=\mathrm{Tz}=\mathrm{z}$

Similarly, since $\{A, S\}$ are weakly compatible then they commute at their coincidence point q. i.e, ASq $=\mathrm{SAq}$ or $\mathrm{Az}=\mathrm{Sz}$.

Now we show that $\mathrm{z}$ is a fixed point of A. By using (4.1.2),

$$
\left\{\begin{array}{c}
{\left[F_{A z, z}(k u)\right]^{2},\left[F_{A z, z}(u)\right]^{2}, F_{A z, A z}(u) F_{z, z}(u), F_{A z, z}(u) F_{A z, A z}(u), F_{A z, z}(u) F_{z, z}(u),} \\
\left.F_{A z, z}(u) F_{A z, z}(2 u), F_{A z, z}(u) F_{z, A z}(u), F_{A z, z}(2 u) F_{z, A z}(u), F_{A z, A z}(u) F_{z, A z}(u),\right\} \geq 0 \\
F_{A z, z}(2 u) F_{z, z}(u)
\end{array}\right\} \geq
$$

$\left[F_{A z, z}(u)\right]^{2} \geq 0$ which means that $\mathrm{Az}=\mathrm{Sz}=\mathrm{z}$.

Thus $\mathrm{z}$ is a common fixed point of $\mathrm{A}, \mathrm{B}, \mathrm{S}$ and $\mathrm{T}$. Finally in order to prove the uniqueness of $\mathrm{z}$, suppose that $\mathrm{z}, \mathrm{w}$ are common fixed points of $\mathrm{A}, \mathrm{B}, \mathrm{S}$ and $\mathrm{T}$. We prove the converse by putting $\mathrm{x}=\mathrm{z}, \mathrm{y}=\mathrm{w}$ in (4.1.2).

$$
\begin{aligned}
& \left\{\begin{array}{c}
{\left[F_{A z, B w}(k u)\right]^{2},\left[F_{S z, T w}(u)\right]^{2}, F_{S z, A z}(u) F_{T w, B w}(u), F_{S z, T w}(u) F_{S z, A z}(u),} \\
F_{S z, T w}(u) F_{T w, B w}(u), F_{S z, T w}(u) F_{S z, T w}(2 u), F_{S z, T w}(u) F_{T w, A z}(u),
\end{array}\right\} \geq 0 \\
& F_{S z, B w}(2 u) F_{T w, A z}(u), F_{S z, A z}(u) F_{T w, A z}(u), F_{S z, B w}(2 u) F_{T w, B w}(u) \\
& \left\{\begin{array}{c}
{\left[F_{z, w}(k u)\right]^{2},\left[F_{z, w}(u)\right]^{2}, F_{z, z}(u) F_{w, w}(u), F_{z, w}(u) F_{z, z}(u), F_{z, w}(u) F_{w, w}(u),} \\
F_{z, w}(u) F_{z, w}(2 u), F_{z, w}(u) F_{w, z}(u), F_{z, w}(2 u) F_{w, z}(u), F_{z, z}(u) F_{w, z}(u), \\
F_{z, w}(2 u) F_{w, w}(u),\left[F_{z, w}(u)\right]^{2}
\end{array}\right\} \geq 0
\end{aligned}
$$

\section{References}

[1] P. Balasubramaniam, S. Murlisankar and R.P. Pant, Common fixed points of four mappings in a fuzzy metric space, J. Fuzzy Math. 10(2) (2002) $379\{384$

[2] M. Grabiec, Fixed points in fuzzy metric space, Fuzzy Sets and System 27 (1988) $385\{389$.

[3] A. George and P. Veermani, On some results in fuzzy metric spaces, Fuzzy Sets and System 64 (1994) $395\{399$.

[4] O. Kramosil and J. Michelak, Fuzzy metric and statistical metric spaces, Kybernetika 11 (1975) $336\{344$.

[5] S. N. Mishra, N. Sharma and S. L. Singh, Common ${ }^{-}$xed points of maps on fuzzy metric spaces, Int. J. Math. Math. Sci. 17 (1994) $253\{258$. 
[6] U. Mishra, A. S. Ranadive and D. Gopal, Fixed point theorems via absorbing maps, Thai J. Math. 6(1) (2008) $49\{60$.

[7] U. Mishra, A. S. Ranadive and D. Gopal, Some Fixed point theorems in fuzzy metric space, Tamkang J. Math. 29(4) (2008) $309\{316$.

[8] V. Popa, Fixed point for non-surjective expansion mappings satisfying an implicit relation, Bul. Sitint. univ. Baia Mare Ser. B Fasc. Mat. Inform 18 (2002) $105\{108$

[9] A. S. Ranadive, D. Gopal and U. Mishra, On some open problems of common - xed point theorems for a pair of non-compatible self-maps. Proc. of Math. Soc., B.H.U. Vol. 20 (2004) $135\{141$.

[10] B. Singh, and S. Jain , Semi-compatibility and ${ }^{-}$xed point theorem in fuzzy metric space using implicit relation, Int. J. Math. Math. Sci. $2005: 16(2005) 2617\{2619$

[11] B. Schwizer and Skalar, Statistical metric spaces, Paci- c J. Math. 10 (1960) $313\{334$

[12] R. Vasuki, Common Fixed point for R-weakly commuting maps in fuzzy metric spaces, Indian J. Pure Appl. Math. 30(4) (1999) $419\{423$

[13] L. A. Zadeh, Fuzzy sets, Information and Control 89 (1965) $338\{353$. 